\title{
Europäisches Strafrecht als Instrument europäischer Integration?
}

\section{Einführung}

Es war wahrscheinlich der Bereich des Strafrechts, der in den letzten Jahren die dramatischste Entwicklung aller EU-Tätigkeitsbereiche erfahren hat. Eine der interessantesten Entwicklungen ist die zunehmend gerichtliche Einmischung in den Bereichen der Dritten Säule. Die nationalen Gerichte (meistens die höheren Instanzen) entschieden Fälle im Zusammenhang mit Fragen des europäischen Strafrechts ${ }^{1}$, doch es waren hauptsächlich die Urteile des Europäischen Gerichtshofs, die hier die meiste Aufmerksamkeit genossen haben $^{2}$. Im folgenden Beitrag wird der Schwerpunkt auf zwei Urteile des EuGH, beide aus dem Umweltstrafrecht, Rechtssachen C-176/03 ${ }^{3}$ und C-440/05 ${ }^{4}$, gelegt. Beide stehen in Verbindung mit verfassungsrechtlichen Fragen im Zusammenhang mit der EU - nämlich dem Kompetenzkonflikt in Strafsachen zwischen dem ersten und dem dritten Pfeiler. Dieser Konflikt bringt weit reichende Folgen im Hinblick auf die Kompetenzverteilung von Europäischer Union und Europäischen Gemeinschaften mit sich. Wir werden versuchen zu analysieren, wie die Urteile des EuGH im strafrechtlichem Bereich den so genannten »Kampf der EU Säulen « (battle of the pillars) beeinflusste und entschied. ${ }^{5}$

\section{Die Zuständigkeit der EG im Bereich des Strafrechts}

Die Debatte über die strafrechtliche Zuständigkeit der EG wird schon seit einiger Zeit geführt. Der Hauptgrund, warum die Debatte bezüglich dieses Problems bis zu den so eben genannten Urteilen ziemlich offen war, ergibt sich daraus, dass der EG Vertrag das Thema Strafrecht nicht als eines der Ziele der EG in Betracht zieht. Wenn das Strafrecht erwähnt wird, dann nur bezüglich der Bereiche in denen die EG keine Handlungsermächtigung hat. ${ }^{6}$ Das Strafrecht gilt als Kernelement der nationalen Sou-

1 Ein gutes Beispiel bieten die Rechtssachen bezüglich des Europäischen Haftbefehls des Bundesverfassungsgerichts (2 BvR 2236/04, 18. Juli 2005), des Obersten Gerichtshofs von Zypern (7. November 2005) und dss Polnischen Verfassungstribunals (P 1/05, 27. April 2005).

2 Die bekanntesten Fällen bleiben aber die folgenden Rechtssachen: Europäischer Haftbefehl Fall C-303/05 (3. Mai 2007), Pupino Fall C-105/03 (16. Juni 2005), und der Umwelt-Fall C-176/03 (13. September 2005).

3 Rechtssache C-176/03 13. September 2005 Kommission der Europäischen Gemeinschaften gegen Rat der Europäischen Union, AB1. C 315,vom 10. Dezember 2005.

4 Rechtssache C-440/05, 23. Oktober 2007 Kommission der Europäischen Gemeinschaften gegen Rat der Europäischen Union, AB1. C 315 vom 22. Dezember 2007.

5 Wasmeier/Thwaites, »The battle of pillars «: does the European Community have the power to approximate national criminal laws?, 29 European Law Review, (2004) S. 613-635.

6 Zum Beispiel Artikel 280 EG-Vertrag ».....Die Anwendung des Strafrechts der Mitgliedstaaten und ihre Strafrechtspflege bleiben von diesen Maßnahmen unberührt. « Siehe auch Artikel 135 EG-Vertrag. 
veränität und die Mitgliedstaaten sind sehr zögerlich, wenn es darum geht, diese aufzugeben. Dies erklärt sich durch die besondere Sensibilität, die dem Gleichgewicht zwischen Individualrechten und dem Staat zugrunde liegt und die nationaler Kultur und Rechtstradition vorbehalten ist sowie aufgrund der strafrechtlichen Natur, welche den Kern der staatlichen Souveränität bildet. ${ }^{7}$

In der Literatur können wir bezüglich der Frage, ob die EG Befugnisse im strafrechtlichen Bereich hat, zwei diametral entgegengesetzte Ansätze finden ${ }^{8}$.

(1) Der erste Ansatz argumentiert, dass die EG nicht auf juristische Disziplinen wie Straf-, Zivil- oder Verwaltungsrecht, sondern auf der Grundlage von politischen Inhalten, wie sie Artikel 3 EGV auflistet, errichtet ist. Diese materiellen politischen Inhalte können jede Art von Reaktion einschließen, auch die des Strafrechts. Die Befürworter dieses Argumentes behaupten, dass es keine Veranlassung gibt, das Strafrecht nicht als eines der EG-Werkzeuge zu verwenden, wenn es notwendig sei, um die EG-Rechtsordnung zu bewahren. Es ist daher nicht erforderlich, das Strafrecht ausdrücklich als eines der Mittel zu erwähnen, mit deren Hilfe Ziele des Gemeinschaftsrechts erreicht werden können. Folglich kann die Gemeinschaft auch Befugnisse in diesem Bereich haben.

(2) Der zweite Ansatz betrachtet das Strafrecht als einen besonderen Fall. Es wurde als Kompetenz nicht ausdrücklich der EG übertragen. Wegen seiner besonderen Sensibilität kann und darf man eine solche Übertragung auch nicht als implizit gegeben ansehen. Nach dem Prinzip der begrenzten Ermächtigung (Artikel 5 EGV) hat die Gemeinschaft nur das Recht, »innerhalb der Grenzen der ihr in diesem Vertrag zugewiesenen Befugnisse und gesetzten Zielen« tätig zu werden. Diese Argumente gewannen nach dem Vertrag von Maastricht noch an Durchschlagskraft. Der dritte Pfeiler basierte auf ganz anderen Prinzipien als der erste. So war es eine Kompetenz der EU, mittels Rahmenbeschlüssen eine Annäherung der Gesetzgebung in Strafsachen zu erreichen. ${ }^{9}$ Dagegen schweige der EG-Vertrag noch immer zu diesem Thema.

Ebenso ist bekannt, verdient aber immer noch hervorgehoben zu werden, dass der Unterschied in der Wahl der Pfeiler oder mit anderen Worten, das Strafrecht als Gegenstand gemeinschaftsrechtlicher oder unionsrechtlicher Verfügungsgewalt, nicht nur eine Frage des Prestiges ist. Vielmehr gibt es auch erhebliche Unterschiede zwischen den Säulen bezüglich des Entscheidungsprozesses, der rechtlichen Wirkung der Maßnahmen und der Rolle der EU-Institutionen. ${ }^{10}$

7 C. Stefanou, European Criminal Law and European Integration Theory, European Journal of Law Reform, VIII/2-3/2007, S. 331.

8 Siehe z.B. Sevenster, Criminal Law and EC Law, 29 Common Market Law Review, (1992) S. 29-64, Wasmeier/Thwaites, The >battle of pillars $<$ does the European Community have the power to approximate national criminal laws? 29 European Law Review (2004) S. 613635, Peers, EU Justice and Home Affairs Law, Oxford University Press, Oxford, 2007.

9 Siehe Artikel 29 und 34 Vertrag der Europäischen Union.

10 Im allgemeinen kann man sagen, dass ein Rechtsinstrument aus der dritten Säule, den Mitgliedstaaten mehr Befugnis einräumt, während ein interinstitutionelles Verfahren aus der ersten Säule eher die Kommission begünstigt. Siehe: Wasmeier/Thwaites, The >battle of pillars $<$ does the European Community have the power to approximate national criminal laws? 29 European Law Review (2004) S. 615. 


\section{Rechtssache C-176/03 (Kommission der EG gegen Rat der EU)}

Wir werden uns jetzt auf den bekannten Fall vom 13. September 2005 fokussieren. Die entscheidende Frage des Falles war: welche strafrechtlichen Befugnisse haben die EU und die EG? Oder anders ausgedrückt: wie ist die Verteilung strafrechtlicher Zuständigkeit zwischen dem ersten und dem dritten Pfeiler?

\section{Der Sachverhalt}

Am 15. März 2001 wurde ein Richtlinienvorschlag des europäischen Parlamentes und des Rates zum Schutz der Umwelt durch das Strafrecht eingebracht ${ }^{11}$ - auf der Rechtsgrundlage von Artikel 175 EG-Vertrag. ${ }^{12}$ Am 27. Januar 2003 erließ der Rat den Rahmenbeschluss zum Schutz der Umwelt durch das Strafrecht ${ }^{13}$ auf der Rechtsgrundlage von Artikel 29, 31 (e) und 34 (2) (b) EUV. Beide, der Vorschlag der Richtlinie und der Rahmenbeschluss, legten eine Reihe von Umweltstraftaten fest, für die die Mitgliedstaaten verpflichtet waren, strafrechtliche Sanktionen vorzusehen. In den Artikeln 1 bis 7 des Rahmenbeschlusses werden verschiedene Handlungen aufgelistet, die als Umweltstraftaten angesehen werden und für die die Mitgliedstaaten strafrechtliche Sanktionen verhängen sollen. ${ }^{14}$ Bei manchen Strafen ging man sogar soweit, dass im schlimmsten Fall eine Freiheitsstrafe angedroht werden sollte. Bezüglich der Sanktionen sieht der Rahmenbeschluss vor, dass jeder Mitgliedstaat die erforderlichen Maßnahmen treffen soll, um sicherzustellen, dass die Teilnahme an oder die Anstiftung zu den genannten Handlungen strafbar ist (Artikel 4). Es geht jedoch nicht darum, die Art der Strafe anzugeben. Jedoch forderte Artikel 5(1) des Rahmenbeschlusses, dass die festgelegten Sanktionen wirksam, angemessen und abschreckend sein sollen und dass »zumindest in schwerwiegenden Fällen auch Freiheitsstrafen umfasst werden, die zur einer Auslieferung führen können «. »Die Strafen können mit anderen Sanktionen oder Maßnahmen einhergehen« (Artikel 5 Abs. 2).

Die Kommission sprach sich in verschiedenen Organen gegen die Rechtsgrundlage des Rahmenbeschlusses aus und behauptete, dass der EG-Vertrag die geeignete Grundlage sei und nicht der EU-Vertrag (Artikel 175 EG-Vertrag anstelle von Artikel 29,31(e) und 34(2)(b) EU-Vertrag). Sie behauptete, dass der Rahmenbeschluss nicht

11 KOM 2001/0139, AB1 2001 C 180 E vom 26. Juni 2001.

12 Dies war keineswegs der einzige Versuch der Kommission, Maßnahmen vorzuschlagen, welche die Mitgliedstaaten verpflichten sollten bei verschiedenen Handlungen Sanktionen zu verhängen. Ähnliche Versuche erfolgten mit der Richtlinie zur Durchsetzung der Rechte des geistigen Eigentums, die die Mitgliedstaaten auffordert, besonders schwere Zuwiderhandlungen solcher Rechte zu bestrafen. (Art. 20, KOM (2003) 46, 30. Januar 2003), Geldwäsche-Richtlinie und die Richtlinie über Marktmissbrauch (Art. 1(1) KOM (2004) 448, 30 Juni 2004.) usw. Siehe Peers, EU Justice and Home Affairs Law, Oxford University Press, Oxford, 2007, S. 391.

13 2003/80/JI, AB1 2003 L 29.

14 Artikel 2, der den Titel trägt »Vorsätzlich begangene Straftaten«, sieht die Merkmale der sieben Straftaten vor, die durch das nationale Recht der Mitgliedstaaten bestraft werden sollten (insbesondere das rechtswidrige Betreiben einer Fabrik, in der eine gefährliche Tätigkeit durchgeführt wird, welches außerhalb dieser Fabrik den Tod oder eine schwere Körperverletzung einer Person oder erhebliche Schäden hinsichtlich der Luft-, Boden- oder Wasserqualität sowie an Tieren oder Pflanzen verursacht oder zu verursachen geeignet ist). 
das geeignete Rechtsinstrument sei, um Umweltstraftaten zu regeln, da der Zweck und der Inhalt dieses Rahmenbeschlusses sich im Geltungsbereich der Gemeinschaft befinden (Titel 9 EG-Vertrag). Sie vertrat die Auffassung, dass es daher nicht der EU obliege, die Mitgliedstaaten aufzufordern, strafrechtliche Sanktionen einzuführen, sondern dass die EG »dafür zuständig (sei), die Mitgliedstaaten zu verpflichten, auf nationaler Ebene Sanktionen - gegebenenfalls auch strafrechtlicher Art - zu verhängen, wenn sich dies als erforderlich erweist, um ein Gemeinschaftsziel zu erreichen $\ll(C-176 / 03) .{ }^{15} \mathrm{Sie}$ forderte den Rat auf, aus dem Rahmenbeschluss ein die Richtlinie im Bereich des strafrechtlichen Schutzes der Umwelt ergänzendes, allein auf die justizielle Zusammenarbeit bezogenes Instrument zu machen und von der Verabschiedung des Rahmenbeschlusses abzusehen, bis die vorgeschlagene Richtlinie angenommen worden sei.

Es ist interessant zu beobachten, dass die EG zwar anerkennt, keine allgemeine strafrechtliche Zuständigkeit zu haben, aber immer noch behauptet, sie dürfe die Mitgliedstaaten verpflichten, strafrechtliche Sanktionen zur Bekämpfung der Verstöße gegen die Umweltschutzbestimmungen vorzusehen, um sicherzustellen, dass die Rechtsvorschrift wirksam ist. Sie selbst behauptet, dass ihr das Recht zustehe, die nationalen Strafrechtssysteme zu harmonisieren - dies gilt insbesondere für die Tatbestandsmerkmale der Umweltdelikte, an die sich strafrechtliche Sanktionen anschließen (Rz. 29). Die Kommission räumt ein, dass es in diesem Bereich keinen Präzedenzfall gebe. Sie verweist jedoch auf die Rechtssprechung des Gerichtshofes zur Loyalitätspflicht und zu den Grundsätzen der Effektivität und der Gleichwertigkeit (Rz. 20).

Der Rat der Europäischen Union behauptet, dass die EG nicht befugt sei, von den Mitgliedstaaten zu verlangen, strafrechtliche Sanktionen hinsichtlich einer im Rahmenbeschluss beschriebenen Tathandlung anzuordnen. In dieser Hinsicht gebe es zwar keine ausdrücklich begrenzte Ermächtigung der Gemeinschaft. Aber wegen der Bedeutung des Strafrechts für die Souveränität der Mitgliedstaaten gebe es auch keinen Grund, warum dieser Tätigkeitsbereich der EG implizit hätte übertragen werden sollen, zumal ihr zugleich spezielle inhaltliche Kompetenzen gemäß Artikel 175 EG-Vertrag verliehen wurden. Diese Behauptung wird vor allem durch die Artikel 135 und 280 EG-Vertrag unterstützt, die den Mitgliedstaaten die Anwendung des nationalen Strafrechts und die gerichtliche Kontrolle ausdrücklich vorbehalten. Ebenso überlässt der EG-Vertrag die Zuständigkeit in Strafsachen ausdrücklich der EU. Der Rat weist auf die Tatsache hin, dass es keine Urteile oder sekundärrechtlichen Vorschriften gibt, die das Argument der Kommission unterstützen. Wann immer die Kommission vorgeschlagen hat, dass die Maßnahmen der Gemeinschaft Auswirkungen auf das Strafrecht haben, griff sie auf die so genannte Praxis der doppelten Gesetzgebung zurück: bei einer Richtlinie oder einer Verordnung im ersten Pfeiler hat der Rat gegebenenfalls den strafrechtlichen Teil abgetrennt und in einen Rahmenbeschluss abgewandelt. ${ }^{16}$

15 Urteil C-176/03 (15).

16 z.B. die Richtlinie zur Definition der Beihilfe zur unerlaubten Ein- und Durchreise und zum unerlaubten Aufenthalt und der Rahmenbeschluss vom 28. November 2002 betreffend der Verstärkung des strafrechtlichen Rahmens für die Bekämpfung der Beihilfe zur unerlaubten Ein- und Durchreise und zum unerlaubten Aufenthalt. 


\section{Die Entscheidung des Gerichtshofes}

Der EuGH entschied, dass der Rahmenbeschluss für nichtig erklärt werden müsse. Mit Bezug auf Artikel 47 EG-Vertrag, der den Vorrang des Gemeinschaftsrechts normiert, sieht der Gerichtshof seine wichtigste Verantwortung darin, sicherzustellen, dass die Handlungen, die in den Anwendungsbereich von Titel 6 EU-Vertrag fallen, nicht in die Zuständigkeiten der Gemeinschaft übergreifen. ${ }^{17}$ Der Umweltschutz ist eines der Hauptziele der EG und da das Ziel und der Inhalt der Artikel 1 bis 7 des Rahmenbeschlusses dem Umweltschutz dienen, können diese Vorschriften wirksam auf der Grundlage des Artikels 175 EG erlassen werden (Rz. 51).

Nach dieser Feststellung weist der Gerichtshof ausdrücklich darauf hin, dass »grundsätzlich das Strafrecht ebenso wie das Strafprozessrecht nicht in die Zuständigkeit der Gemeinschaft fällt« (Rz. 47). Der EuGH führt aus, dass dies die EG aber nicht daran hindert, $»$ Maßnahmen in Bezug auf das Strafrecht der Mitgliedstaaten zu ergreifen, die seiner Meinung nach erforderlich sind, um die volle Wirksamkeit der von ihm zum Schutz der Umwelt erlassenen Rechtsnormen zu gewährleisten, wenn die Anwendung wirksamer, verhältnismäßiger und abschreckender Sanktionen durch die zuständigen nationalen Behörden eine zur Bekämpfung schwerer Beeinträchtigungen der Umwelt unerlässliche Maßnahmen darstellt« (Rz. 48). Im Ergebnis verstieß der ganze unteilbare Rahmenbeschluss gegen Artikel 47 EGV und war daher nichtig.

\section{Diskussion}

Es besteht kein Zweifel, dass der Gerichtshof noch weiterging, als die meisten ProKommissions-Anhänger erwartet hätten. ${ }^{18}$ Grundsätzlich waren sich alle Kommentatoren einig, dass es sich hierbei um eine wegweisende Entscheidung handelte, die wiederum Ausdruck einer aktiven Annäherung des Gerichtshofes ist. Die Entscheidung verwischt endgültig die rechtlichen Unterschiede zwischen den Säulen. Zum ersten $\mathrm{Mal}^{19}$ gibt der EuGH der EG ein Recht, sich in Strafsachen einzumischen. ${ }^{20}$ Der Ge-

17 Siehe ebenfalls Rechtssache C-170/96, Kommission gegen Rat, 12 Mai 1998, Slg. 1998, I2763.

18 So erstaunt die Auffassung nicht, wonach...»it would have been a rash tipster to have advised staking money on the Court of Justice going as far as it did«, Apps, Rechtssache C-176/ 03 Comment, 12 Columbian Journal of European Law, (2005-2006). 625.

19 Es ist keinesfalls das einzige Urteil des Gerichtshofes, das dies zur Folge hat. In gewisser Weise hat der Pupino Fall den Grundsatz der ersten Säule, der unmittelbaren Wirkung schon in der dritten Säule angewendet. Siehe: Mitsilegas, Constitutional Principles of the European Community and European Criminal Law, 8. Eur. J. L. Reform, S. 309-314 (2006). Im Titanium Dioxide Urteil (C-300/89, Slg 1991 I-2867) räumt der EuGH sich das Recht ein, nicht nur die Wahl der Artikel des EG-Vertrags zu beurteilen, sondern auch die Wahl des Pfeilers, S. 628.

20 Wie der Kommentator hinzufügt: «The benefits from the new judgement can be qualified as colossal...« Castillo Garcia, The Power of the European Community to Impose Criminal Penalties, EIPASCOPE, 3, (2005), S 32. 
richtshof versucht tatsächlich, ein Ziel zu erreichen, das schon im gescheiterten Verfassungsvertrag gefordert wurde - nämlich die Vereinigung der drei Säulen.

Der Fall berührt nicht so sehr die Frage nach dem Inhalt dieser Rechtsinstrumente, da sie schon so gut wie identisch sind, als vielmehr die Frage nach dem Niveau der Kontrolle verschiedener Institutionen und der Mitgliedstaaten im Hinblick auf den Bereich des Strafrechts. Oder anders ausgedrückt, es handelt sich um einen »Machtkampf « zwischen der Kommission auf der einen Seite und dem Rat - beziehungsweise den Mitgliedstaaten - auf der anderen Seite. Es geht natürlich um die Frage der unterschiedlichen rechtlichen Auswirkungen, die eine solche Entscheidung mit sich bringt. $^{21}$

\section{Was bedeutet die Behauptung, dass »weder das Strafrecht noch das Strafprozess- recht in die Zuständigkeit der Gemeinschaft «fallen?}

Wir beginnen zunächst mit der Bedeutung des so oft wiederholten Satzes, dass »weder das Strafrecht noch das Strafprozessrecht in die Zuständigkeit der Gemeinschaft « fallen. Oder wie der Generalanwalt es ausdrückt: »Es ist eine gemeinsame Grundüberzeugung, dass das Gemeinschaftsrecht weder eine ausdrückliche noch eine stillschweigende allgemeine Befugnis zur Anordnung von Strafen kennt « (Rz. 27). ${ }^{22}$ Es scheint so, als ob der oft wiederholte Satz, dass »die Gemeinschaft keine Zuständigkeit in Strafsachen hat «, stückweise im Laufe der Zeit seine Bedeutung änderte.

Als erstes werden wir erläutern, was ein solcher Satz rechtlich und sprachlich bedeuten kann.

Die Europäische Gemeinschaft:

- kann die Mitgliedstaaten nur auffordern, effektive punitive Sanktionen vorzusehen, um die Gemeinschaftsziele zu schützen; es obliegt aber den Mitgliedstaaten über die Art und Weise und über den Inhalt dieser Sanktionen zu entscheiden;

- kann einen vergleichbaren Rechtsschutz für nationale Verstöße und Verstöße gegen den EG-Vertrag verlangen;

- kann die Mitgliedstaaten auffordern, strafrechtliche Maßnahmen anzuwenden, einschließlich strafrechtlicher Sanktionen, wenn es für notwendig erachtet wird, die Gemeinschaftsziele zu schützen; sie müssen aber als Rechtsakt des dritten Pfeilers erlassen werden;

- kann Maßnahmen ergreifen, um Mindestvorschriften für die Tatbestandmerkmale strafbarer Handlungen zu schaffen, mit dem Ziel, die Gemeinschaftsziele zu wahren;

- kann Maßnahmen ergreifen, um Mindestvorschriften für die Tatbestandsmerkmale strafbarer Handlungen zu schaffen und die Einführung strafrechtlicher Sanktionen verlangen;

21 Angemerkt sei, dass vor allem dieses Problem verschiedene Mitgliedstaaten während der Debatte um die Europäische Verfassung betraf, da diese wegen der Auswirkungen auf ihre Souveränität besorgt waren. Siehe ein zitiertes Beispiel aus Frankreich im Urteil Nummer 2004-505 vom 19. November 2004 S. 627.

22 Meinung des Generalanwalts Colomer, Rechtssache C-176/03 vom 26. Mai 2005. 
- kann Maßnahmen ergreifen, um Mindestvorschriften im Hinblick auf Tatbestandsmerkmale zu schaffen sowie Art und Umfang der strafrechtlichen Sanktionen vorzuschreiben.

Nach diesen Erläuterungen können wir feststellen, dass der Rat in seiner Vorlage behauptet, dass die Gemeinschaft ein Recht im Sinne der oben genannten Nummer 3 hat, die Kommission hingegen ein Recht im Sinne von Nummer 5 fordert.

Wir können auch zu einer breiteren Perspektive gelangen, indem wir die EuGHRechtsprechung untersuchen. In der Rechtssache Amsterdam Bulb ${ }^{23}$ entschied der EuGH, dass, mangels einer sanktionsbewehrten Vorschrift des Gemeinschaftsrechts, die Mitgliedstaaten befugt sind, die Sanktionen zu wählen, die ihnen sachgerecht erscheinen (Rz. 33). Auch nach Auffassung von Generalanwalt Colomer beruht das Urteil Amsterdam Bulb auf drei Leitgedanken ${ }^{24}$ : (1) Das Gemeinschaftsrecht darf Strafvorschriften (nicht strafrechtliche, sondern nur zivile und verwaltungsrechtliche Vorschriften) einführen, die seine Wirksamkeit schützen, (2) bei Fehlen solcher Vorschriften können die Mitgliedstaaten derartige Mittel, die sie für angebracht halten, anwenden; (3) in diesem Fall steht es ihnen frei, die Modalitäten zu wählen, die ihnen am geeignetsten erscheinen. Der EuGH befürwortet daher das Konzept der oben genannten Nummer 1. Es obliegt der Gemeinschaft, die Mitgliedstaaten aufzufordern wirksame Sanktionen anzuwenden, um das Gemeinschaftsziel zu schützen, aber die Mitgliedstaaten haben das Recht, die Art und Weise dieser Maßnahmen zu bestimmen. Dieselbe Ansicht finden wir im Fall Casati ${ }^{25}$, wo der EuGH entschied, dass die Mitgliedstaaten grundsätzlich für das Strafrecht verantwortlich sind.

Die Entscheidung »Griechischer Mais ${ }^{26}$ fügte zwei Bestimmungen, die die Legitimität nationaler Maßnahmen bei der Umsetzung von EG-Recht betrafen, hinzu:

Verstöße gegen das Gemeinschaftsrecht müssen nach ähnlichen sachlichen und verfahrensrechtlichen Regeln geahndet werden wie nach Art und Schwere gleichartige Verstöße gegen nationales Recht; (2) die Sanktionen müssen wirksam, verhältnismäBig und abschreckend sein (Rz. 24). Der Gerichtshof unterstützte in diesem Fall das oben genannte Konzept Nr. 2: die Gemeinschaft hat nicht das Recht, wegen Verstößen gegen das EG-Recht Sanktionen zu fordern, sie kann aber einheitliche Strafrechtsvorschriften im nationalen und EG-Recht verlangen. Die Mitgliedstaaten müssen Ziele und Rechtsgüter des Gemeinschaftsrechts in gleicher Weise wie eigene Ziele und Rechtsgüter schützen.

In der Rechtssache Nunes und de Matos (1999) ${ }^{27}$ entschied der Gerichtshof, dass die Wahl der Art der Strafe grundsätzlich bei den nationalen Behörden liegt, die Strafen aber vergleichbar sein müssen mit denen, die bei Verstößen gegen innerstaatliche Rechtsvorschriften mit ähnlicher Art und Bedeutung angewendet werden und sie darüber hinaus wirksam, angemessen und abschreckend sein müssen. In dieser Rechtssa-

23 C-50/76 Slg 1977137.

24 Siehe Fußnote 22.

25 Urteil 203/80 Slg 2595.

26 Urteil 68/88 Slg 2965.

27 C-186/98 Slg I-4883. 
che ist zu bemerken, dass das oben genannten Konzept Nummer 2 verfolgt wird - allerdings mit einer Ergänzung im Hinblick auf die Art der angeforderten Strafen: nämlich, die Strafe sollte »wirksam, angemessen und abschreckend« sein. Diese, anfänglich milde, Forderung könnte später dazu führen dass der Gemeinschaft das Recht obliegt, strafrechtliche Maßnahmen und Sanktionen verlangen zu können.

In diesem Zusammenhang ist es sehr lehrreich, das »rhetorische Meisterstïck « von Generalanwalt Colomer in der Rechtssache C-176/03 zu betrachten. Er beginnt seine Analyse der Frage, was eine »wirksame, verhältnismäßige und abschreckende Sanktion « für das Erreichen einer Gemeinschaftsregelung ist, mit zwei Prämissen: Er räumt erstens ein, dass »niemand besser in der Lage ist, die Möglichkeit, Angemessenheit und Wirksamkeit einer strafrechtlichen Reaktion zu beurteilen, als die nationalen Gesetzgeber « (Rz 48), und dass zweitens - trotz des Umstandes, dass die Durchsetzung des Gemeinschaftsrechts in der Verantwortlichkeit der Gemeinschaft liegt - diese Aufgabe (gemeinschaftsrechtswidrige Handlungen zu kriminalisieren) den nationalen Gesetzgebern obliegt « (Rz. 49). Und dann kommt er auf einmal zu dem Schluss, dass für den offenkundigen Fall einer Bestimmung der wirksamen, angemessenen und abschreckenden Strafe es keinen wesentlichen Grund dafür gibt, die Wahl dieser Sanktion nicht demjenigen zu überlassen, der die materielle Zuständigkeit besitzt (Rz. 49). Und für die, die nicht nachvollziehen können, wie er zu dieser Rechtsauffassung gelangt ist, gibt er ein Beispiel, dass die angemessene Sanktion etwa für Angriffe auf das Leben oder den Missbrauch von Minderjährigen Strafcharakter haben muss, so dass, »wenn die bei diesen Verstößen geschützten Rechtsgüter eines der Ziele der Gemeinschaft darstellen, niemand die Befugnis ihrer Rechtsetzungsorgane in Zweifel ziehen würde, von den Mitgliedstaaten die strafrechtliche Verfolgung zu verlangen« (Rz. 50).

Die Verwendung eines solch radikalen Beispiels verwischt das Bild und verleitet den Leser dazu, dem Argument eher nur wegen der Aussagekraft dieses irreführenden Beispiels als wegen der Rechtsgültigkeit dieses Argumentes zuzustimmen. Was der Generalanwalt im Gang seiner Argumente zu vergessen scheint, ist, dass, wenn es keine rechtliche Grundlage gibt, die der Gemeinschaft erlaubt eine Reaktion der Mitgliedstaaten bezüglich des Strafrechts zu verlangen, dies die Gemeinschaft nicht einmal im Falle von Völkermord von den Mitgliedstaaten fordern kann - trotz der Tatsache, dass nur solche Strafen wirksam, verhältnismäßig und abschreckend wären. Wenn die Kriterien so selbstverständlich sind, dann brauchen wir uns wirklich keine Sorgen zu machen, denn die Mitgliedstaaten werden sicherlich die schärfste Waffe benutzen - nämlich das Strafrecht -, um mit einem solchen Verhalten fertig zu werden.

Kurzum, die Einführung der Kriterien »wirksame, verhältnismäßige und abschreckende « Sanktionen öffnen die Tür noch einen Spalt weiter: vielleicht zu einer höheren Ebene als Ebene 2. Weder der Gerichtshof noch das Sekundärrecht ${ }^{28}$ sind je zuvor

28 Die EU-Gesetzgebung verpflichtet die Mitgliedstaaten nur, verschiedene Verhaltensweisen zu bestrafen, überlässt ihnen aber die Wahl der Art und Weise der Strafe. Wie wir wissen verlangte die jüngste Richtlinien-Praxis eine »wirksame, verhältnismäßige und abschrekkende Sanktion«, aber die strafrechtliche Natur dieser Strafen ist im Rahmenbeschluss vorgesehen. 
über das in Nummer 2 erwähnte Modell hinausgegangen, wonach es nicht zu der Zuständigkeit der Gemeinschaft gehört, strafrechtliche Sanktionen für Verstöße gegen das EG-Recht zu verlangen, sie jedoch bei Verstößen gegen das EG-Recht ein gleiches Schutzniveau wie bei Verstößen gegen nationales Recht einklagen kann. Darüber hinaus drückt sich das Gericht so aus: Der Rat und seine Streithelfer vertreten zu Recht die Auffassung, dass die Rechtsprechung keineswegs ausdrücklich eine Gemeinschaftsbefugnis anerkenne, von den Mitgliedstaaten zu verlangen, dass sie Handlungen, die die Verwirklichung der in den Verträgen festgelegten Ziele beeinträchtigen, als Straftaten qualifizieren ( $\mathrm{Rz} 38$ ). Es ist nicht verwunderlich, dass dieses Urteil als eine Überraschung angesehen wird, da der EuGH der Gemeinschaft zumindest ein Modell nach Nummer fünf einräumt. Für das Konzept nach Nummer 6 bleibt die Entscheidung noch ungewiss.

\section{Strafrecht als Mittel zum Zweck}

Eine andere Frage, die zu analysieren ist, ist die kontinuierliche Instrumentalisierung des Strafrechts durch die EU. Anders ausgedrückt - der EuGH gebraucht das Strafrecht als Mittel zum Zweck und nicht als einen bestimmten Rechtsbereich, wo besondere Regeln angewendet werden müssen. ${ }^{29}$ Was meinen wir damit? Das Strafrecht wurde als Mittel zum Zweck wirksamen Schutzes von EG-Standards interpretiert. Der Gerichtshof sieht daher das Strafrecht nicht als einen bestimmten Rechtsbereich an, der auf Prinzipien gegründet ist, die staatliche Macht begrenzen, sondern er überträgt die allgemeinen wirtschaftlichen Grundsätze auf eine Disziplin, deren Sinn und Zweck das genaue Gegenteil ist. ${ }^{30}$

Aufgrund seiner besonderen Natur hätte das Strafrecht nicht implizit auf die EG übertragen werden können. Die Ansicht einer impliziten Befugnis, wie sie von der Kommission vertreten wird, ist eine Auffassung, die es den Strafrechtlern kalt den Rücken hinunterlaufen lässt (Mitteilung der Kommission an das europäische Parlament und den Rat, §7 Rechtssache 440/05, Rz. 29, 36).

Was Spezifika im Strafrecht bedeuten, ist bekannt, aber wir werden es in aller Kürze wiederholen.

(1) Das Strafrecht ist die juristische Disziplin mit den schlimmsten Folgen für die Integrität des Einzelnen. Daher verlangt es nach klaren Kompetenzen und nach präzise

29 Mitsilegas, Constitutional Principles of the European Community and European Criminal Law, 8. Eur. J. L. Reform, S. 309-314 (2006), S. 307.

30 Wir stimmen mit denen überein, die argumentieren: »criminal law is a special case and should be treated differently from economic law «, Mitsilegas, Constitutional Principles of the European Community and European Criminal Law, 8. Eur. J. L. Reform, S. 302. Insbesondere im Lichte des stets genutzten Instrumentariums, das auf Rechtsdurchsetzung/ Sicherheitsaspekte fokussiert, sind die Sorgen um die Wahrung der Beschuldigtenrechte wichtig. (Ebd. 313-314) Leider hat die EU wiederholt ihre Fähigkeit zur Einigung über strafrechtliche Rechtsdurchsetzung und Sicherheitsmassnahmen viel schneller bewiesen als die gleiche Fähigkeit im Hinblick auf Verfahrensgarantien des Beschuldigten. 
formulierten und sorgfältig ausgearbeiteten Vorschriften im materiellen Strafrecht und im Strafprozessrecht. ${ }^{31}$

(2) Die Befugnis zu strafen ist ein wesentliches Merkmal eines souveränen Staates ${ }^{32}$ und die Bedingungen nach denen die Staaten diese Befugnis teilweise übertragen können, müssen ihrerseits eine klare Rechtsgrundlage haben. ${ }^{33}$

(3) Da das Strafrecht ein Rechtsgebiet ist, in dem die Beteiligten am unterschiedlichsten sind (auf der einen Seite der Staat und auf der anderen Seite der einzelne Bürger), muss ein sorgfältiges Gleichgewicht zwischen den Rechten des Staates und denen des Beschuldigten gewährleistet werden (Waffengleichheit/ Grundsatz der Fairness).

(4) Die meisten Maßnahmen im Strafrecht verletzen die Menschenrechte des Beschuldigten und häufig auch die der anderen Bürger. Da die ständige Gefahr besteht, dass diese Maßnahmen fragwürdig und rechtlich zweifelhaft sind, müssen sie durchweg auf einer klaren und vorhersehbaren Rechtsgrundlage aufgebaut sein (Gesetzlichkeitsprinzip).

31 In diesem Punkt können noch viele kritische Anmerkungen hinzugefügt werden im Hinblick auf die Art und Weise, wie das europäische Strafrecht verfasst wird: in aller Schnelle und impulsiv, ohne über die Notwendigkeit nachzudenken, oft unter dem politischen Druck der Staaten oder anderer Interessengruppen, abgesehen von den Warnungen der Menschenrechtsorganisationen usw. Siehe Vermeulen, Criminal policy aspects of the EU's (Internal) asylum policy. Revue des Affaires européennes, Law \& European Affairs, no. 5, (2001/02), pp. 602-612 and - ك̌ ugman/Jager, Post 9/11 Developments of the EU criminal law-related initiatives and their implications on some basic criminal law principles. In: Duyne, P. C., Crime business and crime money in Europe: the dirty linen of illicit enterprise. Nijmegen, Wolf, 2007, S. 247-267.

32 Es gibt noch ein weiteres oft zitiertes Argument, warum die strafrechtlichen Befugnisse nicht an eine supranationale Autorität übertragen werden sollen: das Strafrecht wird oft als Produkt der Kultur bezeichnet, verwurzelt in den Traditionen und Werten einer bestimmten Gemeinschaft. Obschon dieses Argument der Wahrheit entspricht, finden wir nicht, dass dieses Argument wirklich kritisch genug ist, um zu rechtfertigen, weshalb das Strafrecht nicht an die EU übertragen werden sollte. Es entspricht auch der Wahrheit, dass, als wir uns bereit erklärt haben in einem grenzenlosen Europa zu leben, wir damit auch unser Einverständnis gaben verschiedene Bereiche der staatlichen Souveränität aufzugeben. Oder wie es van der Wilt hinzufügt: »In its crudest form, [this argument] ... seems to ignore the fact that States may be inclined to sacrifice some of their uniqueness for the common good. After all, this is the essence of the whole process of economic, political and cultural integration in Europe. It is by no means self-evident that this process should stop at the door of criminal law. A more serious objection is that this essentially conservative approach may obstruct the necessary measures which would enable the European States to face the challenges raised by organised crime «, van der Wilt, Some critical reflections on the process of harmonisation of criminal law within the European Union, in: Harmonization and Harmonizing Measures in Criminal Law, Klip/van der Wilt (Hrsg.), Royal Netherlands Academy of Arts and Sciences, Amsterdam, 2002, S. 77-78.

33 Dies erklärt, warum das Strafrecht ziemlich spät Einzug in einen der Politikbereichen des EG-Vertrages fand und warum ein spezieller Pfeiler errichtet wurde, um solch sensible Fragen zu handhaben. Der dritte Pfeiler, als der Ausdruck der Zurückhaltung in den Mitgliedstaaten bezüglich der Einmischung der EG in diesen Bereichen, basiert auf anderen Prinzipien wie die Zusammenarbeit zwischen den Behörden und nicht auf supranationalen Grundsätzen der Gemeinschaft. 
(5) Da das Strafrecht ein solch mächtiges Instrument ist, sollte es nur als ultima ratio verwendet werden; ${ }^{34}$

(6) dies ist der Grund, weshalb nach diesen universalen Strafrechtsprinzipien die Interventionen des Staates (oder einer supranationalen Behörde) nur möglich sind, wenn sie durch das Gesetz ausdrücklich und detailliert formuliert sind - sie können nicht implizit aus einem Rechtsakt abgeleitet werden.

Daher ist es für einen Strafjuristen schwierig zu akzeptieren, dass strafrechtliche Befugnisse einer bestimmten Institution implizit auf eine andere Institution übertragen werden könnten, insbesondere da diese Institutionen ihre Handlungen nicht immer auf Strafrechtsprinzipien gründen. In diesem Fall wurde das Strafrecht als Steuerungsinstrument genutzt. Die Ziele, die man erreichen will, gehorchen indes ganz anderen Grundsätzen (denen des Binnenmarkts), wenn man die Regeln und Prozeduren zur Annahme strafrechtlicher Befugnisse nicht im Voraus innerhalb der Mitgliedstaaten vereinbart. Dies trifft sogar auf einer allgemeinen Ebene zu, ganz zu schweigen davon, wenn wir anfangen, die bekannten Argumente des Rates aufzuzählen, die er zur Unterstützung seiner Behauptung anführte (zum Beispiel, dass der ganze Titel 6 des EUVertrags auf ganz anderen Prinzipien der intergouvernementalen Zusammenarbeit aufbaut).

\section{Gerichtsurteil $C-440 / 05$}

Nach dem ersten Urteil C-176/03 blieben einige wichtige Fragen in Bezug auf die strafrechtlichen Befugnisse der EG unbeantwortet. Die erste noch offene Frage war, was die genauen Kriterien für die Festlegung der strafrechtlichen Kompetenzen der EG sind: Sind die strafrechtlichen Befugnisse der EG nur auf den Umweltschutz erweitert worden oder bezieht sich dieselbe Logik auf andere Gemeinschaftsziele und wenn ja, welche? Die zweite unbeantwortete Frage bezieht sich auf den Anwendungsbereich der EG-Befugnisse im strafrechtlichen Bereich: Hat die EG nur das Recht, Mindestanforderungen an Straftatbestände festzulegen oder kann sie die Art, und vielleicht sogar die Höhe der Strafe bestimmen?

Die Kommission legte ihre Ansicht in ihrer Mitteilung an das Europäische Parlament und den Rat dar ${ }^{35}$ : sie macht geltend, dass dieselben Argumente aus der Rechtsprechung auf andere gemeinsame Politiken und Grundfreiheiten angewendet werden können (Freizügigkeit von Personen, Waren-, Dienstleistungs-, und Kapitalverkehr). Dennoch gibt sie zu, dass das Strafrecht keine EG-Politik bildet, sie aber nach den Erläuterungen des EuGH in der Rechtssache C-176/03 das Strafrecht nutzen kann, um Integrationsziele zu erreichen, jedoch nur unter der Bedingung, dass dieses Vorgehen

$34 \breve{S}$ ugman/Jager, Post 9/11 developments of the EU criminal law-related initiatives and their implications on some basic criminal law principles. In: Duyne, P. C. (Hrsg.), Crime business and crime money in Europe: the dirty linen of illicit enterprise. Nijmegen, Wolf, 2007, S. 247-267.

35 Mitteilung der Kommission an das Europäische Parlament und den Rat über die Folgen des Urteils Gerichtshofs vom 13 September 2005 (Rs. C-176/03, Kommission gegen Rat) /* KOM/2005/0583 endg. */. 
sowohl notwendig als auch kohärent ist. Sie gelangt zu der Schlussfolgerung, dass im Rahmen eines Beschlussvorschlags die Erforderlichkeit einer strafrechtlichen Intervention im Einzelfall geprüft werden muss. Dann ist sie in der Lage, zum einen die Merkmale der Straftaten zu definieren und zum anderen Art und Maß der anzuwendenden Sanktionen festzulegen. Demnach könnte es sein, dass die Freiheit der Mitgliedstaaten, selbst strafrechtliche Sanktionen festzulegen, durch die EG eingeschränkt wird. Schließlich folgert die Kommission, dass der Praxis der doppelten Gesetzgebung ein Ende gesetzt wird: es gibt keinen Unterschied mehr zwischen Richtlinie und Rahmenbeschluss.

Der EuGH hingegen kommt in seinem Urteil C-440/05 zu einer etwas anderen Auffassung. In dieser Rechtssache finden wir - verglichen mit dem vorangegangenen Urteil (C-176/03) - eine Wiederholung und eine Abweichung. Die Kommission stellt einen Aufhebungsantrag im Hinblick auf den Rahmenbeschlusses des Rates 2005/667/JI vom 12. Juli 2005 (Meeresverschmutzung durch Schiffe). ${ }^{36}$ Dieser Rahmenbeschluss war im Hinblick auf die Anwendung strafrechtlicher Maßnahmen dem vorhergehenden sehr ähnlich, ging aber zugleich auch einen Schritt weiter. Neben der Tatsache, dass er Mindestvorschriften von Straftatbeständen festlegt, beschreibt er nicht nur die Art der strafrechtlichen Sanktion, sondern auch das Maß. So sieht Artikel 4 Abs. 1 vor: »Jeder Mitgliedstaat ergreift die erforderlichen Maßnahmen, um sicherzustellen, dass Straftaten nach Artikel 2 oder 3 mit wirksamen, angemessenen und abschreckenden strafrechtlichen Sanktionen bedroht sind, die zumindest in schweren Fällen auch Freiheitsstrafen im Höchstmaß von mindestens einem bis drei Jahren umfassen «.

Die Kommission war der Ansicht, dass das Ziel und der Inhalt dieses Rahmenbeschlusses in den Zuständigkeitsbereich der EG fiele (nämlich der gemeinsamen Verkehrspolitik) und dass das Kriterium der Erforderlichkeit erfüllt sei. So müsse der EuGH wie in der Rechtssache C-176/03 entscheiden. Sie wiederholte den öfters erwähnten Satz, die EG habe eine strafrechtliche Annexkompetenz, obwohl das Strafrecht nicht als eigener Politikbereich der Gemeinschaft ausgewiesen sei. Der Rat behauptete, dass die Verkehrspolitik kein so wesentliches, übergreifendes und grundlegendes Ziel sei wie der Umweltschutz. Demnach könne der Gerichtshof eine andere Ansicht vertreten und dem Rat das Recht gewähren, strafrechtliche Vorschriften zu erlassen. Der Rat vertrat ebenfalls das Argument, dass die Ansicht des Gerichtshofs in der Rechtssache C-176/03 eher eine Ausnahme und nicht die Regel war.

Der EuGH schlussfolgerte, dass Artikel 80 EG-Vertrag keine konkreten Einschränkungen im Hinblick auf die Natur der Rechtsakte, die der Rat annehmen kann, vorsieht und dass die Gemeinschaft eine weit reichende Gesetzgebungsbefugnis in diesem Bereich hat. Da der Rahmenbeschluss sich auf die Verhaltensweise bezieht, welche aller Wahrscheinlichkeit nach zu besonders schweren Umweltschäden führen kann, ist eine strafrechtliche Annäherung unerlässlich für die Sicherheit auf See und den Umweltschutz. Daher müsse man strafrechtlich vorgehen. Da der Inhalt des Rahmenbeschlusses auf der Grundlage des EG-Vertrags angenommen werden kann, müsse der Rahmenbeschluss für nichtig erklärt werden. Der Gerichtshof entschied den

36 AB1 2005 L 255. 
Fall wieder auf der Grundlage von Artikel 47 - Prinzip des Vorrangs des Gemeinschaftsrechts. Jedoch hebt der Gerichtshof dann hervor, dass » die Bestimmung von Art und Maß der anzuwendenden strafrechtlichen Sanktionen (...) dagegen, anders als die Kommission meint, nicht in die Zuständigkeit der Gemeinschaft« falle (Rz. 70). Also, die Art und das Maß der Strafen fallen nicht in den Zuständigkeitsbereich der EG.

Der Gerichtshof unterstreicht noch einmal, dass nicht nur der Umweltbereich so wichtig ist, dass der EG eine strafrechtliche Intervention erlaubt ist, sondern dass diese auch auf andere Gemeinschaftsziele ausgeweitet werden kann, wenn das Erforderlichkeitskriterium erfüllt ist. Die Zuständigkeit der EG bezieht sich indes nur darauf, Mindestvorschriften der Straftatbestände zu bestimmen und strafrechtliche Sanktionen zu fordern, aber nicht die Art und das Maß der Strafe zu bestimmen. Dieses fällt in den Zuständigkeitsbereich der EU.

\section{Die scheinbare Schlussfolgerung und die unbegründete »Erforderlichkeit»}

Beide Urteile werfen die kriminalpolitische Frage der vernünftigen Mittel-Zweck-Relation auf. Wenn dies tatsächlich zutrifft, dann ist die Anwendung der »wirksamen, angemessenen und abschreckenden strafrechtlichen Sanktion « eine wesentliche und notwendige Maßnahme, »um die volle Wirksamkeit der von ihm zum Schutz der Umwelt erlassenen Rechtsnormen zu gewährleisten« (C-176/03 Rz. 48). Oder anders ausgedrückt: unter welchen Umständen müssen wir auf strafrechtliche Sanktionen zurückgreifen, um eine wirksame Politik zu erreichen? Im Zusammenhang mit einer behaupteten Mittel-Zweck-Rationalität scheint dies eine empirische Frage zu sein.

Mit dem Konzept der vollen Wirksamkeit einer Politik zu beginnen, ist problematisch, weil es nicht klar genug und daher als Maßstab des Zwecks ungeeignet ist. Wenn man dieses Konzept idealiter als Maßstab betrachtet, so könnte es einen vollen Erfolg dieser Politik und die vollständige Beseitigung eines Problems zum Gegenstand haben. In der Rechtssache C-176/03 gerät der Gerichtshof in einen Zirkelschluss, wenn er behauptet, dass die EG befugt sei, strafrechtliche Sanktionen anzuwenden, um »die volle Wirksamkeit der von ihr zum Schutz der Umwelt erlassenen Rechtsnormen zu gewährleisten, wenn die Anwendung wirksamer, verhältnismäßiger und abschreckender Sanktionen (...) eine zur Bekämpfung schwerer Beeinträchtigungen der Umwelt unerlässliche Maßnahme darstellt«. Anders ausgedrückt, es ist der EG erlaubt, das Strafrecht anzuwenden, wenn sie der Auffassung ist, dass es eine wesentliche Bedeutung im Umgang mit der Umweltkriminalität hat. Oder einfacher ausgedrückt, die EG ist befugt, das Strafrecht anzuwenden, wenn es sich um Verbrechen handelt - das ist nun wirklich tautologisch. Schließlich stützt der Gerichtshof seine Entscheidung noch nicht einmal ansatzweise auf empirische Erwägungen, die seine letztlich empirischen Annahmen stützten könnten. Er behauptet lediglich, dass das Strafrecht als »letzter Ausweg « angewendet werden soll.

Auch Generalanwalt Colomer glaubt, dass in diesem Fall die Erforderlichkeit strafrechtlicher Sanktionen auf der Hand liege und vergleicht diesen Fall mit der offensichtlich selbstverständlichen Anwendung des Strafrechts in Fällen der Tötung 
Minderjähriger oder von Korruption (Rz. 50). Das Problem dieser Art von Schlussfolgerungen ist, dass die Richter und Juristen in der Regel schlecht beraten sind, um diese Frage der potenziellen Wirksamkeit verschiedener politischer Maßnahmen in abstracto zu beurteilen. Insbesondere im Fall von umweltschädlichen Aktivitäten könnte eine tiefergehendere Analyse im Hinblick auf die behauptete Wirkung des Strafrechts eine bessere Grundlage bieten, als sie eine Perspektive ex cathedra vorsehen kann.

Dies gilt umso mehr, als das Problem, wie Umweltschäden durch den Gebrauch wirksamer politischer Instrumente wirklich verhindert werden können, erhebliches wissenschaftliches Interesse auf sich zieht. So sehen zum Beispiel Bowles, Faure und Garoupta das Strafrecht nur als eines der Instrumente an, um umweltschädliche Aktivitäten zu kontrollieren. Es konkurriert mit anderen Alternativen wie dem Zivil- und Verwaltungsrecht, mit der Zusammenarbeit von Privaten oder mit Verbrauchssteuern - alles Mittel zur Prävention von Umweltschäden. Der erforderliche und geeignete Anwendungsbereich des Strafrechts ist demnach ganz pragmatisch durch eine KostenNutzen-Frage bestimmt. Erforderlich ist jedenfalls eine genaue empirische Untersuchung, um die beste institutionelle Lösung innerhalb eines bestimmten Kontextes zu gewährleisten. ${ }^{37}$

Daher sollten Behauptungen, dieses oder jenes politische Instrument sei selbstverständlich und offensichtlich notwendig, tunlichst vermieden werden - um vorsichtig zu formulieren. Der Gerichtshof hat nicht die notwendigen Mittel, um die komplexe Frage des zur Erreichung von Gemeinschaftszielen wirklich geeigneten Instruments zu entscheiden. Leider hat der Gerichtshof - weder in der Rechtssache C-176/03 noch in der Rechtssache C-440/05 - eine klare Leitlinie gefordert oder entwickelt, wann strafrechtliche Maßnahmen wirklich erforderlich sind. Er hat einfach die Stellungnahme der Kommission übernommen. ${ }^{38}$

Zugleich ist sich die Kommission des Problems offensichtlich bewusst und versucht, die Anwendung des Strafrechts in einem konkreten Fall durch eine tiefgründigere Analyse zu rechtfertigen. Im Falle des Umweltschutzes wurde dies durch eine Folgenabschätzung der Kommission getan und zwar mit dem Begleitdokument zum »Vorschlag für eine Richtlinie des Europäischen Parlaments und des Rates über den strafrechtlichen Schutz der Umwelt «. ${ }^{39}$ Dieses Dokument enthält den einzigen vernünftigen Vorschlag, nämlich, dass es verschiedene politische Optionen und Möglichkeiten im Kampf gegen schwere Umweltschäden gibt. Danach bewertet und beurteilt

37 Bowles/Faure/Garoupa, Why Criminal Law, paper presented on EALE Conference, Ljubljana, Slovenia, September 2005, S. 14. Sie führen weiter aus und schließen daraus, dass $» \ldots$ the detailed structure of a mix of fiscal, regulatory and legal provisions may be used in an effort to internalise an externality. Finding the cost minimising combination, including the most appropriate role (if there is to be one) for criminal law, requires empirical investigation of the incentive structure facing participants in the activity. Ibidem, S. 29.

38 Siehe K.M. Rechtssache C-176/03, Kommission gegen Rat: »Pillars Askew: Criminal Law EC-style«, 12 Colum. J. Eur. L., (2005-2006) S. 29.

39 Siehe: http://ec.europa.eu/governance/impact/docs/ia_2007/sec_2007_0160_en.pdf. Siehe ebenfalls Leitlinien für die Folgenabschätzung (15. Juni 2005 mit der März Version), Europäische Kommission SEC (2005) 791. 
sie gründlich eine nach der anderen. Es ist jedoch unglücklich, dass diese Studie der Folgenabschätzung wieder demselben, bereits beschriebenen Zirkelschluss anheim fällt. Bei der ersten Konfrontation mit dem Problem, was bezüglich der Umweltkriminalität zu tun ist, kam sie zu dem Schluss, dass die strafrechtliche Antwort als politische Option von wesentlicher Bedeutung sei, dass also Kriminalität wesentlich nach einer strafrechtlichen Antwort verlangt.

Gleichwohl: in der Welt der Zweck-Mittel-Rationalität bei der Wahl eines wirksamen politischen Instrumentes, stellen das Verfassen und die öffentliche Präsentation der Studie der Folgenabschätzung den einzig richtigen Weg dar, der eingeschlagen werden kann. Solche Studien fokussieren sich auch auf schwerwiegende Probleme. Aber die Erfahrungs- und Forschungsansichten stehen im direkten Gegensatz zu den Erklärungen über die Verwendung des Strafrechts als offensichtlich selbstverständlichem politischen Instrument. Das Problem der Argumentation des EuGH in dem wegweisenden Urteil C-176/03 und anschließend in der Rechtssache C-440/05 ist, dass der Gerichtshof die offensichtliche Erforderlichkeit des Strafrechts nicht vorrangig mit seiner symbolischen Wirkung begründet, die - wenn überhaupt - in diesem Fall selbstverständlich wäre, sondern als das im Rahmen einer Zweck-Mittel-Relation wirklich erforderliche politische Instrument. Dies erfolgte zwei Jahre zuvor in der Rechtssache C-176/03, bevor die Studie zur Folgenabschätzung von der Kommission fertig gestellt und im Jahre 2007 der Öffentlichkeit zugänglich gemacht wurde.

\section{Fazit}

Grundsätzlich kann man sich dem Gedanken nicht verschließen, die Umwelt auch mit den Mitteln des Strafrechts zu schützen. Dies gilt um so mehr, wenn man zu dieser Schlussfolgerung nach einer umfassenden Diskussion über die Rolle und die Funktion des Strafrechts gelangt ist. Aber als Juristen, insbesondere als Strafrechtler, benötigen wir als erstes eine gültige Rechtsgrundlage. Es wäre nicht vorstellbar gewesen, dass das Strafrecht ohne weiteres - implizit - der EG hätte übertragen werden können und es fällt schwer, die Auffassungen des Gerichtshofes zu rechtfertigen, der mehr als aktiver Fortbilder des Gesetzes, mehr als Verfechter der Kommissionspolitik denn als Justizinstanz das Recht auslegt. Er überdehnt den strafrechtlichen Bereich als Gegenstand des ersten Pfeilers ${ }^{40}$, ohne eine ausdrückliche Billigung seitens der Mitgliedstaaten zu haben. Wenn die Mitgliedstaaten ihre strafrechtlichen Kompetenzen an die EG hätten übertragen wollen, dann hätten sie das ausdrücklich und nach vielen Verhandlungen, die wir derzeit öfters in der dritten Säule erleben, getan.

Diese ganze Diskussion scheint im Lichte des neuen Vertrags von Lissabon, der nach Ratifikation in allen 27 Mitgliedstaaten - die Unterschiede zwischen den Säulen abschafft, nutzlos zu sein. Zugleich ist es dennoch sehr aufschlussreich, die Auffassung und die Rolle des Gerichtshofes und die Art, wie die EU-Institutionen die traditi-

$40 »$ The judgment therefore amounts in practice to a >transfer of a part of the third pillar to the first pillar...«, S. Peers, EU Justice and Home Affairs Law, Oxford University Press, Oxford, 2007, S. 393. 
onelle Auffassung und die Grundprinzipien des Strafrechts übergehen, zu untersuchen. Das Gericht hat erneut eine aktive Rolle angenommen und sich damit über den Willen der Mitgliedstaaten und die Grundprinzipien des Strafrechts hinweggesetzt. Dies ist sicherlich kein gutes Zeichen für das künftige Europa. 